\section{Effect of the microfiltration process on antioxidant activity and lipid peroxidation protection capacity of blackberry juice}

\author{
Gabriela Azofeifa, ${ }^{1}$ Silvia Quesada, ${ }^{* 1}$ Ana-Mercedes Pérez ${ }^{2}$ \\ ${ }^{1}$ Departamento de Bioquímica, Escuela de Medicina, Universidad de Costa Rica, \\ ${ }^{2}$ Centro Nacional de Tecnología de Alimentos, Universidad de Costa Rica.
}

\begin{abstract}
Phytochemicals are highly concentrated in berries, especially polyphenols as anthocyanins and ellagitannins. These compounds have been associated with antioxidant capacity, lipid peroxidation protection, anti-inflammatory activity, anticarcinogenic activity, obesity prevention and others. Blackberries are commonly grown and consumed as juice in Latin-American countries. However, blackberry juice is easily fermented and different industrial techniques are being applied to enable the juice to be stored for longer periods. One important issue required for these techniques is to preserve the health-promoting capacities of blackberries. This study compared the antioxidant activity and the lipid peroxidation protector effect between a fresh blackberry juice (FJ) and a microfiltrated blackberry juice (MJ). Chemical analysis of both juices show less polyphenols concentration in the MJ. Despite this difference, values for biological activities, such as protection of lipid peroxidation, was not significantly different between FJ and MJ. These results suggest that the compounds responsible for the antioxidant activity are maintained even after microfiltration and the free radical scavenging capacity of these compounds could protect the initiation of lipid peroxidation. Microfiltration could be used as an industrial technique to produce blackberry juice that maintains biological activities of polyphenols.
\end{abstract}

Revista Brasileira de Farmacognosia Brazilian Journal of Pharmacognosy 21(5): 829-834, Sep./Oct. 2011

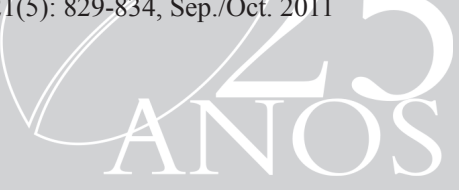

Article

Received 27 Dec 2010

Accepted 22 Apr 2011

Available online 5 Aug 2011

Keywords:

antioxidant capacity

blackberry juice

lipid peroxidation microfiltration

Rubus adenotrichus

ISSN 0102-695X http://dx.doi.org/10.1590/S0102$695 \times 2011005000133$

\section{Introduction}

Several studies have suggested a potential health benefit of flavonoids (Beattie et al., 2005; He \& Giusti, 2010). Particularly, there has been documented an inverse association between high consumption of flavonoids and the incidence of cardiovascular diseases (Mazza, 2007). Phytochemicals, such as flavonoids, can act as free radical scavengers or can up regulate endogenous antioxidant defenses. Some flavonoids suppress reactive oxygen species formation, by chelation of transition metal ions or by the suppression of superoxide-driven reactions that generate active oxygen species (Pietta, 2000).

Compared with most fruit, berries have high concentrations of a type of flavonoids known as anthocyanins (Beattie et al., 2005). Anthocyanins have been associated also with antioxidant activities, lipid peroxidation protection, anti-inflammatory activity, anticarcinogenic activity, obesity prevention and others (He \& Giusti, 2010).

Blackberries are promoted as rich sources of polyphenols with antioxidant activities (Jiao \& Wang, 2000; Acosta-Montoya et al., 2010). Blackberry juice consumption demonstrates the capacity to increase the plasma antioxidant capacity in humans (Hassimotto et al., 2008). Moreover, purified anthocyanin fractions of blackberry demonstrated anti-inflammatory activity by inhibition of COX-1 and COX-2 enzymes (Rossi et al., 2003; Cuevas-Rodriguez et al., 2010; Bowen-Forbes et al., 2010).

Major phenolic compounds in the blackberry variety Rubus adenotrichus Schltdl., Rosaceae, are anthocyanins cyanidin 3-glucoside and cyanidin 3-(6'malonyl) glucoside and ellagitannins lambertianin $\mathrm{C}$ and sanguiin H-6 (Mertz et al., 2007). Both types of molecules were associated as main contributors to the antioxidant capacity in another berry Rubus idaeus L., Rosaceae, (Mullen et al., 2002).

Juice processing conditions cause differences in antioxidants content (Gil et al., 2000). Industrial processing with the addition of sugar and pectin; thermal treatments; and long term storage, can have marked effects on the phenolic content of berries, sometimes causing the increase of some compounds and the decrease of others (Zafrilla et al., 2001; Gancel et al., 2010).

According to Mullen et al. (2002), in raspberries these effects are more important in ellagitannins than in anthocyanins; however there is no effect in the antioxidant 
capacity of the fruit. Gil et al. (2000) described that pomegranate hand press juices and commercial juices had the same anthocyanin pigments, but quantitative differences. Pomegranate anthocyanins are partially degraded or transformed into other products due to freezing procedures prior to juice extraction.

Some publications have studied changes in blackberries anthocyanins properties due to processing, storage and heat treatments. Cisse et al. (2009) explained that anthocyanins of Rubus adenotrichus are less sensitive to heat when compared to anthocyanins from other fruits and other varieties of blackberry. Hager et al. (2008) reported a decrease in the scavenging activity against peroxyl radicals and superoxide radicals (ORAC and PCL) after thermally processed blackberry products, but not after storage time.

Microfiltration is an application with special importance in fruit-juices. Efforts have been done to produce practical models that can be used for industrial scale and to test parameters that influence the performance of microfiltration techniques (Vaillant et al., 2008). However, it is also important to evaluate the possible effects of the microfiltration technique in beneficial roles documented for fruits-juices rich in polyphenols.

The aim of this study was to evaluate the effect of microfiltration of blackberry juice on the antioxidant capacity and the lipid peroxidation protection effect.

\section{Materials and Methods}

\section{Sample collection}

Samples were prepared with full-ripe blackberries (Rubus adenotrichus Schltdl., Rosaceae, cv. 'vino') harvested in different farms of the province of Cartago, Costa Rica (altitude $1864 \mathrm{~m}-2517$, latitude 09 $39^{\prime} 57.1^{\prime \prime} \mathrm{N}$

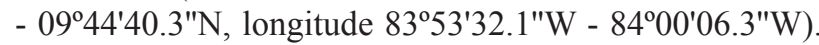
To prepare the fresh juice (FJ), blackberries were blended and filtrated in gauze to reduce suspended solids. The microfiltrated juice (MJ) was prepared according to Vaillant et al. (2008). Blackberries were pressed and the juice treated with a commercial enzymatic preparation (Klerzyme $^{\circledR} 150$ from DSM Food Specialties, Heerlen, Netherlands) for $1 \mathrm{~h}$ at $35^{\circ} \mathrm{C}$ with constant agitation. The microfiltration was perform in a tubular ceramic membrane (Membralox $^{\circledR} 1$ P19-40, Pall Exekia, Bazet, France) with an average pore size diameter $0.2 \mu \mathrm{m}$. Both samples were stored at $-30{ }^{\circ} \mathrm{C}$ until analysis.

\section{Analytical methods}

\section{Moisture content}

The moisture content was determined by the weight difference of the samples after freeze-dried. Each sample was analyzed in duplicate.

\section{Total polyphenol content}

Total phenolic content was determined with Folin-Ciocalteu assay modified by George et al. (2005). Results were expressed as milligrams of gallic acid equivalent (GAE) per gram of dry matter (DM). Each sample was analyzed in four replicates.

\section{Anthocyanin content}

Total anthocyanins content was determined by $\mathrm{pH}$ differential method (Lee et al., 2005b). Briefly, juice (pH 1.0 and 4.5) absorbance was measured at $510 \mathrm{~nm}$ and corrected with the absorbance at $700 \mathrm{~nm}$. The calculation of total anthocyanin content was performed using the molecular weight (449.2 g. $\left.\mathrm{mol}^{-1}\right)$ and molar extinction coefficient of the cyanidin 3-glucoside (26900 L.mol ${ }^{-1}$. $\mathrm{cm}^{-1}$ ). Results were expressed as mg cyanidin 3-glucoside per gram of dry matter (DM). Each sample was analyzed in four replicates.

\section{Antioxidant capacity}

\section{DPPH radical- scavenging activity}

The radical-scavenging activity (RSA) of the fresh juice and the microfiltrated juice were evaluated by assessing their direct DPPH-scavenging activity (HyunJin et al., 2004). DPPH $0.25 \mathrm{mM}$ was prepared in methanol and $0.5 \mathrm{~mL}$ of this solution incubated with $1 \mathrm{~mL}$ of sample dilutions. Mixtures were incubated at room temperature in the dark for $30 \mathrm{~min}$ and the absorbance of DPPH was measured at $517 \mathrm{~nm}$. Methanol $(0.5 \mathrm{~mL})$ plus juice dilution $(1.0 \mathrm{~mL})$ were used as sample blank. Percentage of radical scavenging activity of the juice was calculated according the formula: \%RSA $=[1-(\mathrm{Abs}$ sample/Abs control) $]^{*} 100$. RSA percentage was plotted against the sample concentration and a linear regression curve was established in order to calculate the IC50, which means that amount of juice necessary to reach the $50 \%$ radical scavenging activity. Results were expressed as $\mu \mathrm{g} D \mathrm{DM} /$ $\mathrm{mL}$. Each sample was analyzed in triplicate.

\section{Oxygen radical absorbance capacity (ORAC)}

ORAC assay was performed according to $\mathrm{Ou}$ et al. (2001). Fluorescein was used as fluorescent probe and the oxidation was induced with AAPH (2,2-azobis-2methyl-propanimidamide-dihydrochloride). Assays were performed in spectrofluorimeter equipment (Biotek Instruments). ORAC values were expressed as Trolox equivalents (TE/g DM). 


\section{Protection to lipid peroxidation in liposomes}

Liposomes were prepared according to Pérez et al. (2003). Briefly, $25 \mathrm{mg}$ of commercial lecithin was dissolved in $2.15 \mathrm{~mL}$ of chloroform and then $350 \mu \mathrm{L}$ of methanol was added. This mix was dried using a nitrogen atmosphere and finally the lecithin was resuspended in 4.5 $\mathrm{mL}$ of warm phosphate buffered saline (PBS) and sonicated for $1 \mathrm{~h}$ at $4{ }^{\circ} \mathrm{C}$ to form liposomes.

To test the juice capacity to protect lipid peroxidation, an oxidative stress was induced with AAPH (2,2-azobis-2-methyl-propanimidamide-dihydrochloride). Fifty microliters of juice dilutions were mixed with $0.45 \mathrm{~mL}$ of liposomes and $0.2 \mathrm{~mL}$ of AAPH in a final concentration of $10 \mathrm{mM}$. These solutions were incubated in the dark for $2 \mathrm{~h}$ at $37{ }^{\circ} \mathrm{C}$. To determine malondialdehyde (MDA) concentration, $0.2 \mathrm{~mL}$ of TCA $5 \%$ (trichloroacetic acid) and $1 \mathrm{~mL}$ TBA $0.75 \%$ (thiobarbituric acid) were added and samples were heated at $100{ }^{\circ} \mathrm{C}$ for $30 \mathrm{~min}$. After cooling, $0.25 \mathrm{~mL} \mathrm{SDS} 3 \%$ was added and centrifuged at $4000 \mathrm{rpm}$ for $15 \mathrm{~min}$. The absorbance of the supernatant was measured at $532 \mathrm{~nm}$. MDA concentrations were plotted against the sample concentration and a linear regression curve was established, results were expressed as the amount of juice that inhibits $50 \%$ of lipid peroxidation (IC50).

\section{Protection to lipid peroxidation in liver homogenates}

Sprague-Dawley rats $(220 \pm 20 \quad$ g) were anesthetized and sacrificed by decapitation according to the Institutional Committee for Care and Handling of Experimental Animals of Universidad de Costa Rica (CICUA \# 19-06). Liver tissue of each rat was obtained and homogenized in phosphate buffered saline (PBS) using an Ultraturrax T-25 equipment (Ika-Labortechnik) to obtain a tissue suspension at $20 \%$. The suspension was centrifuged at $9000 \mathrm{x} g$ during $15 \mathrm{~min}$ to reduce suspended solids. Seventy-five microliters of different concentrations of juices were added to $0.75 \mathrm{~mL}$ of liver-supernatant and incubated for $30 \mathrm{~min}$ at $37^{\circ} \mathrm{C}$. Subsequently, an oxidative stress was induced with TBHP (tert-butyl hydroperoxide) in the final concentration of $1.7 \mathrm{mM}$ and incubated for 1 $\mathrm{h}$ at $37^{\circ} \mathrm{C}$. Finally, thiobarbituric acid reactive substances (TBARS) were measured as the end product of lipid peroxidation.

TBARS was assayed according to Uchiyama \& Mihara (1978). Briefly, $0.25 \mathrm{~mL}$ of liver homogenate, $0.25 \mathrm{~mL}$ of $35 \%$ TCA and $0.25 \mathrm{~mL}$ of Tris-HCl buffer $(50$ $\mathrm{mM}, \mathrm{pH} 7.4$ ) were mixed and incubated $10 \mathrm{~min}$ at room temperature. Then, $0.5 \mathrm{~mL}$ of $0.75 \%$ TBA was added and heated at $100{ }^{\circ} \mathrm{C}$ for $45 \mathrm{~min}$. After cooling, $0.5 \mathrm{~mL}$ of $70 \%$ TCA was added, mixed and centrifuged at $4000 \mathrm{rpm}$ for 15 min. The absorbance of the supernatant was measured at $532 \mathrm{~nm}$. Concentration of TBARS was assessed using the molar absorption coefficient for malondialdehyde (MDA) of $1.56 \times 10^{5} \mathrm{~cm}^{-1}-\mathrm{M}^{-1}$ and results were expressed as nmol $\mathrm{MDA} / \mathrm{g}$ liver tissue. MDA concentrations were plotted against the sample concentration and a linear regression curve was established to calculate the IC50.

The assay was performed using liver tissue from five rats. To establish basal levels, MDA levels without TBPH were assessed in each rat. Due to the color of blackberries, sample blanks were prepared in each experiment. Each juice sample was tested in triplicate.

\section{Statistical analysis}

To compare $\mathrm{FJ}$ and $\mathrm{MJ}$ results, statistical analysis was undertaken using ANOVA and Tukey's test. A $p$ value $<0.05$ was accepted as statistically significant.

\section{Results}

Analytical methods

Table 1 shows the moisture content, total polyphenols (TP) and anthocyanins content of the fresh juice and the microfiltrated juice. The amount of total polyphenols and anthocyanins for both samples are significantly different $(p<0.001)$

Table 1. Total polyphenols and anthocyanins content in two blackberry samples.

\begin{tabular}{|c|c|c|c|}
\hline & $\begin{array}{c}\text { Moisture content } \\
\left(\% F W^{-1}\right)\end{array}$ & $\begin{array}{c}\mathrm{TP} \\
m g G A E g^{-1} D M \\
\end{array}$ & $\begin{array}{c}\text { Anthocyanins } \\
\text { mg cyanidin 3-glucoside } \text { g }^{-1} D M\end{array}$ \\
\hline Fresh Juice (FJ) & $92.0 \pm 0.1^{\mathrm{a}}$ & $45.0 \pm 0.5^{\mathrm{a}}$ & $11.5 \pm 0.1^{\mathrm{a}}$ \\
\hline Microfiltrated Juice (MJ) & $90.1 \pm 0.1^{\mathrm{a}}$ & $24.9 \pm 1.0^{\mathrm{b}}$ & $5.6 \pm 0.3^{\mathrm{b}}$ \\
\hline
\end{tabular}

DM, dry matter, TP, total polyphenols, GAE, Gallic acid equivalents. Each value is mean \pm SE of four replicate experiments. Means in columns followed with different letters differed significantly $(\mathrm{p}<0.001)$.

Table 2. Radical-scavenging activity of two blackberry samples.

\begin{tabular}{lcc}
\hline & DPPH IC50 $\left(\mu g D M m L^{-1}\right)$ & ORAC $\left(\mu m o l T E g^{-1} D M\right)$ \\
\hline Fresh Juice (FJ) & $101.2 \pm 4.8^{\mathrm{a}}$ & $547 \pm 10^{\mathrm{a}}$ \\
Microfiltrated Juice (MJ) & $94.08 \pm 4.2^{\mathrm{a}}$ & $417 \pm 11^{\mathrm{b}}$ \\
\hline
\end{tabular}

Each value is mean \pm SE of three replicate experiments. DM, dry matter. Means in columns followed with different letters differed significantly $(p<0.05)$. 


\section{Antioxidant capacity}

The DPPH and ORAC assay were utilized to evaluate the antioxidant capacity of both juices. Table 2 shows the amount of each juice necessary to reach the $50 \%$ radical scavenging activity (DPPH), and for ORAC the $\mu \mathrm{mol}$ of trolox equivalents for each sample. The comparison of values for DPPH do not show significant differences $(p>0.05)$ but for ORAC the values are significantly different $(p<0.05)$.

\section{Protection to lipid peroxidation}

To evaluate protection to lipid peroxidation, lecithin liposomes were incubated with both samples of blackberry juices and exposed to an oxidative stress induced with AAPH. Both juices decrease levels of lipid peroxidation measured as MDA concentration. The IC50 indicates the amount of juice necessary to decrease $50 \%$ of MDA concentration of liposomes treated with AAPH. The IC50 was $155.7 \pm 13.1 \mu \mathrm{g} \mathrm{DM} / \mathrm{mL}$ for MJ and $134.7 \pm 11.0 \mu \mathrm{g}$ $\mathrm{DM} / \mathrm{mL}$ for FJ. These IC50 values do not show significant differences $(p>0.05)$.

Figure 1 shows the inhibitory effect of both blackberry juices against lipid peroxidation induced by TBHP in a liver homogenates of rats. Results indicate a hepatoprotective effect against oxidative stress. FJ and MJ decrease levels of lipid peroxidation in a dose-dependent manner. The amount of FJ and MJ necessary to decrease $50 \%$ of MDA concentration of the control liver tissue treated with TBHP are $462 \pm 72 \mu \mathrm{g} \mathrm{DM} / \mathrm{mL}$ and $429 \pm 118$ $\mu \mathrm{g} \mathrm{DM} / \mathrm{mL}$, respectively. Samples do not show significant differences $(p>0.05)$.

\section{Discussion}

Microfiltration technique to produce juices involves mechanical and enzymatic treatments, transmembrane pressure and elimination of some compounds due to retained particles (Vaillant et al., 2008;
Riedl et al., 1998). All these factors affect polyphenols composition of the final juice. As it is shown in this investigation, the microfiltration process decreases the concentrations of total polyphenols and anthocyanins when it is compared to the fresh juice. Gil et al. (2000) emphasize that in the industrial process to produce juice some phenolic compounds are extracted from the fruit, but depending on the technique other ones could increase.

The antioxidant activity measured by ORAC was significantly different between FJ and MJ but is not significantly different when is measured by DPPH. According to Niki (2010) variations among antioxidant activity measured by DPPH and ORAC are expected because DPPH is based in the reaction with a stable free radical while ORAC is an assessment by a competition method. This distinction causes a different relative contribution of the compounds responsible for the antioxidant activity in each technique.

The results show that the capacity to protect lipid peroxidation is not affected by the differences in the total polyphenol composition of FJ and MJ. This was confirmed both in the liver model and in the liposomes model. It is well known that free radicals are involved to initiate lipid peroxidation (Lee et al., 2005a). Therefore, the high antioxidant capacity shown by FJ and MJ permits an effective radical scavenging and therefore protects to lipid peroxidation. Lee et al. (2005a), suggested that the ability of different compounds to scavenge ROS in liver tissue is particularly important because it's main role in metabolism of exogenous chemicals that usually generate ROS and induce oxidative stress.

In this study, despite ORAC differences for FJ and $\mathrm{MJ}$, the capacity to protect against lipid peroxidation is similar for both samples, even when the lipid peroxidation is induced with AAPH or TBHP. This is in agreement with Niki (2010) who suggests that the capacity of free radical scavenging by antioxidants does not necessarily correlate with the capacity of inhibition of lipid peroxidation. Specifically, this author states that ORAC method does not show the capacity of antioxidants to inhibit lipid peroxidation.

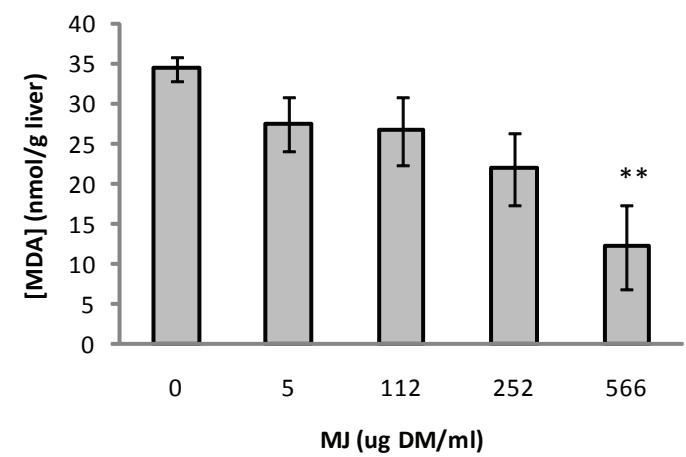

Figure 1. Protective effect of fresh juice (A) and microfiltrated juice (B) on TBHP induced oxidative stress in liver homogenates model. Each value is mean $\pm \mathrm{SE}$ (three independent experiments). ${ }^{*} p<0.05$, ${ }^{* *} p<0.01$ compared with homogenates treated with TBHP. 
This study concludes that differences in the amounts of polyphenols and anthocyanins after the microfiltration could alter the antioxidant capacity but do not alter the capacity to protect against lipid peroxidation. Mullen et al. (2002) evaluated changes in polyphenols quantity due to industrial process with similar results to our study. Mullen's study in raspberries evaluates the effect of short-term treatments, such as storage and freezing. For raspberries, significant differences in total phenolics were observed and changes between free and conjugated forms of anthocyanins were evident. Mullen concluded that antioxidant capacity is not due to a single anthocyanin, instead it is a combined contribution of different anthocyanins.

Blackberry juice has a very short shelf-life and that is why microfiltration process coupled to freezing storage is important to maintain the juice available for longer periods. Several other industrial processes are available, but some of them such as pasteurization present an important reduction of the health-promoting properties of processed-juice (Gancel et al., 2010). Microfiltration technique coupled to UHT packaging provides a sterile juice thanks to the pore used in the membranes. This study suggests that this procedure could permit the preservation of the capacity to inhibit lipid peroxidation in the microfiltrated juice. However, further research is needed to characterize the compounds, the interactions of polyphenols after the microfiltration process, and how these affect biological activities.

\section{Acknowledgment}

Financial support for this work was funded by Vicerrectoría de Investigación, Universidad de Costa Rica (Project No. 422-A7-028), Producing Added-Value from Under-Utilized Crops project (PAVUC-FP6-INCO project- DEV-2, contract 015279). Authors thank Marielos Torres (CITA, UCR) and AL Torres (Departamento de Bioquímica, Escuela de Medicina, UCR) for their technical assistance.

\section{References}

Acosta-Montoya O, Vaillant F, Cozzano S, Mertz C, Perez AM, Castro MV 2010. Phenolic content and antioxidant capacity of tropical highland blackberry (Rubus adenotrichus Schltdl.) during three edible maturity stages. Food Chem 119: 1497-1501.

Beattie J, Crozier A, Duthie GG 2005. Potential health benefits of berries. Curr Nutr Food Sci 1: 71-86.

Bowen-Forbes CS, Yanjun Z, Muraleedharan GN 2010. Anthocyanin content, antioxidant, anti-inflammatory and anticancer properties of blackberry and raspberry fruits. J Food Compos Anal 23: 554-560.

Cisse M, Vaillant F, Acosta O, Dhuique-Mayer C, Dornier M 2009. Thermal degradation kinetics of anthocyanins from blood orange, blackberry, and roselle using the Arrhenius, Eyring, and Ball models. J Agric Food Chem 57: 6285-6291.

Cuevas-Rodriguez EO, Díaz VP, Yousef GG, García PA, López J, Paredes O, Gonzalez E, Lila MA 2010. Inhibition of pro-inflammatory responses and antioxidant capacity of Mexican blackberry (Rubus spp.) extracts. J Agric Food Chem 58: 9542-9548.

Gancel AL, Feneuil A, Acosta O, Pérez AM, Vaillant F 2010. Impact of industrial processing and storage on major polyphenols and the antioxidant capacity of tropical highland blackberry (Rubus adenotrichus). Food Res Int (In Press) doi:10.1016/j.foodres.2010.06.013.

George S, Brat P, Alter P, Amiot MJ 2005. Rapid determination of polyphenols and vitamin $\mathrm{C}$ in plant-derived products. J Agric Food Chem 53:1370-1373.

Gil MI, Tomás FA, Hess B, Holcroft DM, Kader AA 2000. Antioxidant activity of pomegranate juice and its relationship with phenolic composition and processing. J Agric Food Chem 48: 4581-4589.

Hager TJ, Howard LR, Prior RL 2008. Processing and storage effects on monomeric anthocyanins, percent polymeric color, and antioxidant capacity of processed blackberry products. J Agric Food Chem 56: 689-695.

Hassimotto NMA, Da Silva Pinto M, Lajolo FM2008. Antioxidant status in humans after consumption of blackberry (Rubus fruticosus L.) juices with and without defatted milk. $J$ Agric Food Chem 56:11727-11733.

He J, Giusti M 2010. Anthocyanins: natural colorants with healthpromoting properties. Food Sci Technol 1: 163-187.

Hyun-Jin K, Feng C, Changqoing W, Xi W, Hau YC, Zhengyu J 2004. Evaluation of antioxidant activity of Australian tea tree (Malaleuca alternifolia) oil and its components. $J$ Agric Food Chem 52: 2849-2854.

Jiao H, Wang SY 2000. Correlation of antioxidant capacities to oxygen radical scavenging enzyme activities in blackberry. J Agric Food Chem 48: 5672-5676.

Lee H, Lee HS, Won NH, Kim KH, Jun W, Lee KW 2005a. Antioxidant effects of aqueous extract of Terminalia chebula in vivo and in vitro. Biol Pharm Bull 28: 16391644.

Lee J, Durst W, Wrolstad RE 2005b. Determination of total monomeric anthocyanins pigment content of fruit juices, beverages, natural colorants, and wines by the $\mathrm{pH}$ differential method: collaborative study. J AOAC Int 88 : 1269-1278.

Mazza GJ 2007. Anthocyanins and heart health. Ann Ist Super Sanita 43: 369-374.

Mertz C, Cheynier V, Günata Z, Brat P 2007. Analysis of phenolic compounds in two blackberry species (Rubus glaucus and Rubus adenotrichus) by high-performance liquid chromatography with diode array detection and electrospray ion trap mass spectrometry. J Agric Food Chem 55: 8616-8624.

Mullen W, McGinn J, Lean MEJ, MacLean MR, Gardner P, Duthie GG, Yokota T, Crozier A 2002. Ellagitannins, flavonoids, and other phenolics in red raspberries and their contribution to antioxidant capacity and vasorelaxation properties. J Agric Food Chem 50: 5191-5196.

Niki E 2010. Assessment of antioxidant capacity in vitro and in vivo. Free Radic Biol Med 49: 503-515. 
Ou B, Hampsch-Woodill M, Prior RL 2001. Development and validation of an improved oxygen radical absorbance capacity assay using fluorescein as the fluorescent probe. J Agric Food Chem 49: 4619-4626.

Pérez RM, Vargas R, Martínez FJ, García EV, Hernández B 2003. Antioxidant activity of alkaloids from Bocconia arborea. A study on six testing methods. Ars Pharm 44: 5-21.

Pietta PG 2000. Flavonoids as antioxidants. J Nat Prod 63: 1035 1042.

Riedl K, Girard B, Lencki RW 1998. Influence of membrane structure on fouling layer morphology during apple juice clarification. J Membrane Sci 139: 155-166.

Rossi A, Serraino I, Dugo P, Di Paola R, Mondello L, Genovese T, Morabito D, Dugo G, Sautebin L, Caputi AP, Cuzzocrea S 2003. Protective effects of anthocyanins from blackberry in a rat model of acute lung inflammation. Free Radic Res 37: 891-900.

Uchiyama M, Mihara M 1978. Determination of malonaldehyde precursor in tissues by thiobarbituric acid test. Anal
Biochem 86: 271-278.

Vaillant F, Pérez AM, Acosta O, Dornier, M 2008. Turbidity of pulpy fruit juice: a key factor for predicting cross-flow microfiltration performance. J Membrane Sci 325: 404412.

Zafrilla P, Ferreres F, Tomás-Barberán FA 2001. Effect of processing and storage on the antioxidant ellagic acid derivatives and flavonoids of red raspberry (Rubus idaeus) jams. J Agric Food Chem 49: 3651-3655.

\section{*Correspondence}

\section{Quesada Silvia}

Departamento de Bioquímica, Escuela de Medicina, Universidad de Costa Rica, San Pedro, 2060, San José, Costa Rica.

silvia.quesada@ucr.ac.cr

Tel. +50625114450

Fax: +506 25115338 